\title{
El doble propósito del informe de alta en el hospital "Hermanos Ameijeiras"
}

\section{Dual purpose of the hospital discharge report in "Hermanos Ameijeiras" hospital}

\author{
María del Carmen Alemán Lage'; Norma Eneida Ríos Massabot'"; Francisco \\ Hernández Romero"'I \\ 'Máster en Salud Pública. Especialista de II Grado en Administración de Salud. \\ Hospital Clínico Quirúrgico "Hermanos Ameijeiras". La Habana, Cuba. \\ "Doctora en Ciencias Médicas. Hospital Clínico Quirúrgico "Hermanos Ameijeiras". \\ La Habana, Cuba. \\ "Técnico Básico en Informática. Hospital Clínico Quirúrgico "Hermanos Ameijeiras". \\ La Habana, Cuba.
}

\begin{abstract}
RESUMEN
La necesidad de incluir un mayor número de historias clínicas en el local asignado para archivo y localizar en estos con mayor rapidez la información de valor que se desea de hospitalizaciones anteriores, propició un estudio encaminado a resolver esta dificultad, el que recomendó sustituir la hoja de egreso vigente, por otro formulario diseñado expresamente, denominado Informe de Alta. Se establecieron las normas de trabajo para la extracción de documentos dos años después de concluida la hospitalización del paciente. A partir de enero del año 2000 el hospital "Hermanos Ameijeiras" sustituyó la hoja de egreso por el Informe de Alta y lo incluyó en el proyecto de automatización del departamento de registros médicos del hospital. Después de ocho años de la implantación del Informe de Alta, con un doble propósito: eliminación de documentos de la historia clínica y ser registro básico para obtener información de calidad para la toma de decisiones, se realizó una evaluación que rindió buenos resultados que se presentan en este trabajo.
\end{abstract}

Palabras clave: Informe de alta, el archivo de historias clínicas, indicadores hospitalarios. 


\section{ABSTRACT}

The need of keeping higher number of medical histories in some premises for archiving and of locating as fast as possible the valuable information on previous stays at hospital if needed, prompted the carrying out of a study to solve this difficulty, which recommended to replace the present discharge form by another custom-designed formulary called Hospital discharge report. The working standards to draw documents out two years after the patient hospitalization were set. From J anuary 2000 on, "Hermanos Ameijeiras" hospital replaced the discharge form by the Discharge Report and included it in the automation project of the medical history department. After eight years of Discharge Report implementation with two objectives: elimination of documents from the medical histories and creation of a basic register to get quality information for decision-making, the evaluation presented in this paper yielded good results.

Key words: Discharge report, medical history archives, hospital indicators.

\section{NTRODUCCI ON}

La historia clínica es el documento donde queda constancia de todos los procesos relacionados con la salud de los pacientes y donde de forma directa o indirecta se refleja el esfuerzo diario de todos los que trabajan en un hospital. ${ }^{1}$

El sistema de historia clínica única es indudablemente el más correcto, sin embargo, junto a la tendencia de dejar una constancia cada vez más amplia por escrito respecto de cada admisión, crea sus propios problemas. A medida que el paciente recibe más atención ingresado, el documento se engrosa, lo que trae aparejado dos problemas: primero, se hace más difícil de manipular y ordenar, por lo que se pierde mucho tiempo a la cabecera del enfermo y se pueden extraviar detalles esenciales en la masa de papeles (lo que resulta ser un elemento fundamental), segundo, el local necesario para dar cabida a estos documentos en su totalidad (y la organización que se necesita para archivar, indexar, identificar y recuperar las historias) debe ser cada vez más amplio. ${ }^{2}$ El almacenamiento de los informes de alta digitalizados soluciona este problema. ${ }^{3}$

En un estudio anterior del departamento de estadística del hospital "Hermanos Ameijeiras", ${ }^{4}$ se propuso un sistema para la eliminación selectiva de historias clínicas que implicaba la eliminación de documentos a los dos años para lo cual era necesario el llenado de un modelo llamado Informe de Alta (IA).

El IA confeccionado se ensayó en el hospital y se aplicó en el año 2002. Al cierre temporal del hospital en el año 2004, este modelo fue revisado, haciéndose las adaptaciones y estableciéndose los requisitos para su aplicación automática, (recogida en el $\mathrm{SIH}$ ) de tal forma que además del impacto que se esperaba que tuviera en el archivo de historias clínicas, sirviera de base para la confección de indicadores hospitalarios y de servir como elemento de referencia del hospital con la atención primaria. El modelo fue aplicado a partir de recomenzar las hospitalizaciones en el centro en el año 2005. 
Al cabo de tres años de la revisión del modelo, se ha querido evaluar el impacto de este formulario.

\section{EL I NFORME DE ALTA}

El resumen de egreso fue sustituido por el IA, lo llena el médico que cierra la historia clínica de esa hospitalización y es el responsable de la calidad de los datos anotados. Permanece en la historia clínica hasta la eliminación de esta del archivo; las instrucciones para su llenado aparecen escritas en el propio formulario (anexo).

Los datos se incluyen en el registro automatizado de la forma siguiente: El número de historia clínica y los datos generales del paciente lo capta la Sección de Admisión, al anotar el número de identidad del paciente del registro de inscripciones del hospital, a su vez, los datos sobre los servicios en que fue hospitalizado se incorporan automáticamente del movimiento hospitalario.

Al egreso del paciente y en paquetes organizados por día de egreso (para mayor rapidez) se introducen los códigos de las afecciones que fueron tratadas y los procedimientos médicos empleados (quirúrgicos y no quirúrgicos), así como el estado al egreso y de forma automática aparece la descripción de cada código para su revisión inmediata. El nombre y número de registro del médico, lo toma del Registro de profesionales previamente configurado.

El resumen electrónico de cada paciente derivado del IA contiene los datos generales, el motivo de ingreso (codificado), las causas de egreso codificadas (principal y secundarias), la fecha de ingreso y egreso, los procedimientos realizados durante la hospitalización y las complicaciones si las hubo. Al mismo tiempo se cuenta con todos los datos de los procedimientos quirúrgicos, codificados y clasificados por tipo de operación, cirujano, anestesia, así como queda el registro electrónico del médico que egresa al paciente.

Este resumen automatizado de la historia se puede solicitar e imprimir con rapidez y corresponderá a una hospitalización determinada o a todas las hospitalizaciones de pacientes que su historia clínica aún esté en el archivo o no, ya que el mismo nunca se elimina.

\section{EL I NFORME DE ALTA PARA EL ARCHIVO}

El archivo del hospital se rige por las normas nacionales que indican: ${ }^{5}$

- Sistema de numeración única.

- Eliminación del archivo de historias clínicas de los pacientes cuya última hospitalización ocurrió hace 5 años.

El hospital utiliza el sistema de numeración única, pero no se asigna al paciente un número consecutivo por orden de llegada como expresa la norma, se utiliza como número de historia clínica el número de identidad del paciente, este último tiene como ventaja que el porcentaje de pacientes con más de una historia clínica en el archivo es muy pequeño. Esta deficiencia sólo puede ocurrir en pacientes que 
ingresan por urgencia sin carné de identidad, a los que se les asigna un número provisional y en ninguna oportunidad posterior se conoció su número de identidad.

El número provisional que se le asigna a los cubanos y el que se les asigna a los extranjeros consta de 11 caracteres igual al número de identidad, con la diferencia que el primero tiene un carácter alfabético que indica que se trata de un provisional para cubano y el segundo, 2 caracteres alfabéticos que informan el país de procedencia. Ambos son conformados de forma automática a partir de la fecha de nacimiento y el sexo del paciente y prevé que la cantidad de historias clínicas que se incorporan a cada una de las secciones y divisiones del archivo sea igual. Los tres tipos de número cuentan con un algoritmo de chequeo que impiden que las diferentes secciones que incorporan información a un paciente le asigne la que le corresponde a otro.

El número de historia clínica que se utiliza desvincula a esta con la fecha en que se originó, lo que imposibilita el cumplimiento de las normas de cómo organizar el grupo de candidatos a pasivo y eliminación de historias después de 5 años de la última hospitalización. ${ }^{5}$

Este hecho ocasionaba que fuera más laboriosa la eliminación de historias clínicas, por lo que nunca se realizó esta actividad de forma sistemática antes de junio de 2004, fecha en que cerró la institución para reparación.

La automatización del IA permite obtener listas diarias de pacientes egresados cualquiera que sea el tiempo atrás, sean cubanos o extranjeros. Esto facilita que el archivo conozca los listados de pacientes que su última hospitalización ocurrió hace 2 y 5 años, así como los pacientes extranjeros que su última hospitalización ocurrió en el tiempo que se establezca para este tipo de paciente. De la primera lista se obtienen las historias clínicas de las que es necesario extraer documentos de la última hospitalización. De las otras dos, las historias clínicas que se deben eliminar del archivo. ${ }^{4,5}$

Una vez recomenzado el trabajo del archivo en el 2006, se procedió a la depuración selectiva y continua de las hojas de la historia a los $2 \operatorname{años}^{4}$ y a la depuración en el papel a los 5 años ${ }^{5}$ quedando para ambos casos un resumen de historia clínica electrónica.

EI IA facilita este trabajo continuo. La documentación en cada hospitalización al cabo de 2 años se reduce de un promedio de 20 a 5 hojas, o sea el $74 \%$ de reducción por historia.

Actualmente el archivo cuenta con información de 213631 hospitalizaciones, de ellas 62109 con la documentación completa correspondiente a los egresos de los últimos 2 años: 2004 y 2005. El resto, 151522 hospitalizaciones fueron depuradas quedando con el IA y los documentos señalados con la palabra "GUARDAR" por los médicos, dicho de otro modo, el $71 \%$ de los documentos tienen como promedio 5 hojas, lo que sin dudas frena el crecimiento del archivo.

A las historias clínicas que regresan al archivo procedentes de la sala, se les realiza la evaluación cuantitativa y en especial el llenado del IA, además, el Comité de Evaluación de Historias Clínicas califica la calidad de los datos que ofrece en especial sobre morbilidad. 


\section{EL I NFORME DE ALTA Y LOS I NDI CADORES}

A partir del IA se obtiene un conjunto de indicadores, estos pueden calcularse para todas las hospitalizaciones del hospital, de un servicio o sala determinada y el período puede ser desde 1 día hasta el máximo de tiempo deseado.

La base de datos del sistema computarizado permite obtener información solamente del IA y en reportes relacionados con la información obtenida durante la inscripción del paciente, el movimiento hospitalario, el informe operatorio y otros.

La morbilidad se obtiene a partir de la lista tabular de cuatro caracteres de la Clasificación Internacional de Enfermedades Décima Revisión (CIE-10), ${ }^{6}$ a la que se le han realizado algunas ampliaciones y creado listas abreviadas específicas para el hospital, iguales adecuaciones se realizaron para el Manual de Procedimientos en Medicina (CIE-9). ${ }^{7}$

A continuación se relacionan los indicadores más utilizados:

- Lista de todos los IA que reúnan los requisitos previamente establecidos, ordenados por la variable que se desee. De estas listas se pueden obtener indicadores para los que no existen reportes específicos.

- Motivo de ingreso. La estructura de los motivos de ingreso se puede conocer por protocolos de actuación.

- Promedio de estadía. Se obtiene por diagnóstico principal, de un diagnóstico o de una o más agrupación diagnóstica que se establezca y preoperatorio de los ingresos electivos.

- Morbilidad por diagnóstico principal. Estructura por edad y sexo y estructura según residencia habitual.

- Morbilidad por diagnósticos coexistentes, por complicaciones durante la hospitalización y causas externas de las hospitalizaciones por causas violentas. Se obtiene la estructura de cada una y las relaciones con un diagnóstico principal.

- Readmisión. Para el cálculo de este indicador, el tiempo máximo a considerar entre la fecha de egreso de la primera hospitalización y la fecha de ingreso de la segunda hospitalización se debe seleccionar en cada oportunidad. De acuerdo a las agrupaciones diagnósticas que se establezcan se pueden obtener readmisiones por complicaciones médicas y quirúrgicas, por tratamientos y otros. Además, es posible obtener listas de pacientes readmitidos con los datos siguientes: servicios en que estuvo hospitalizado, fechas de egresos y diagnóstico principal de cada hospitalización (código y descripción).

- Procedimientos médicos. Estructura según las listas establecidas y combinadas con variables del informe operatorio.

- Mortalidad. Estructura de acuerdo a la lista de morbilidad que se desee, relación porcentual entre el número de fallecidos y el total de egresos por la misma causa y cruces con otras variables que se seleccione.

Finalmente se puede concluir que el Informe de Alta ha permitido la eliminación del máximo de hojas de cada hospitalización. Es posible obtener información sobre las 
hospitalizaciones de un paciente después de eliminada su historia clínica del archivo. La automatización del Informe de Alta eliminó la confección de tarjeteros manuales para la morbilidad y los procedimientos. Los indicadores de morbilidad y procederes médicos se obtienen con mayor rapidez. La evaluación del Informe de Alta por el Comité de Evaluación de Historias Clínicas permite obtener información estadística de calidad.

Se recomienda la sustitución de la Hoja de Egreso por el Informe de Alta para todas las unidades hospitalarias del país, a fin de que esté implementado antes de su automatización. Continuar con las tareas de automatización del Informe de Alta de manera que quede un resumen electrónico de cada hospitalización que ayude además al trabajo y ahorre tiempo médico a esta actividad. Confeccionar los nomencladores que aún faltan para obtener la información necesaria en cada oportunidad. Aumentar de 4 a 10 puntos el correcto llenado del Informe de Alta cuando se realiza la evaluación cualitativa de la historia clínica.

\section{Anexo}

Llenar con letra de molde

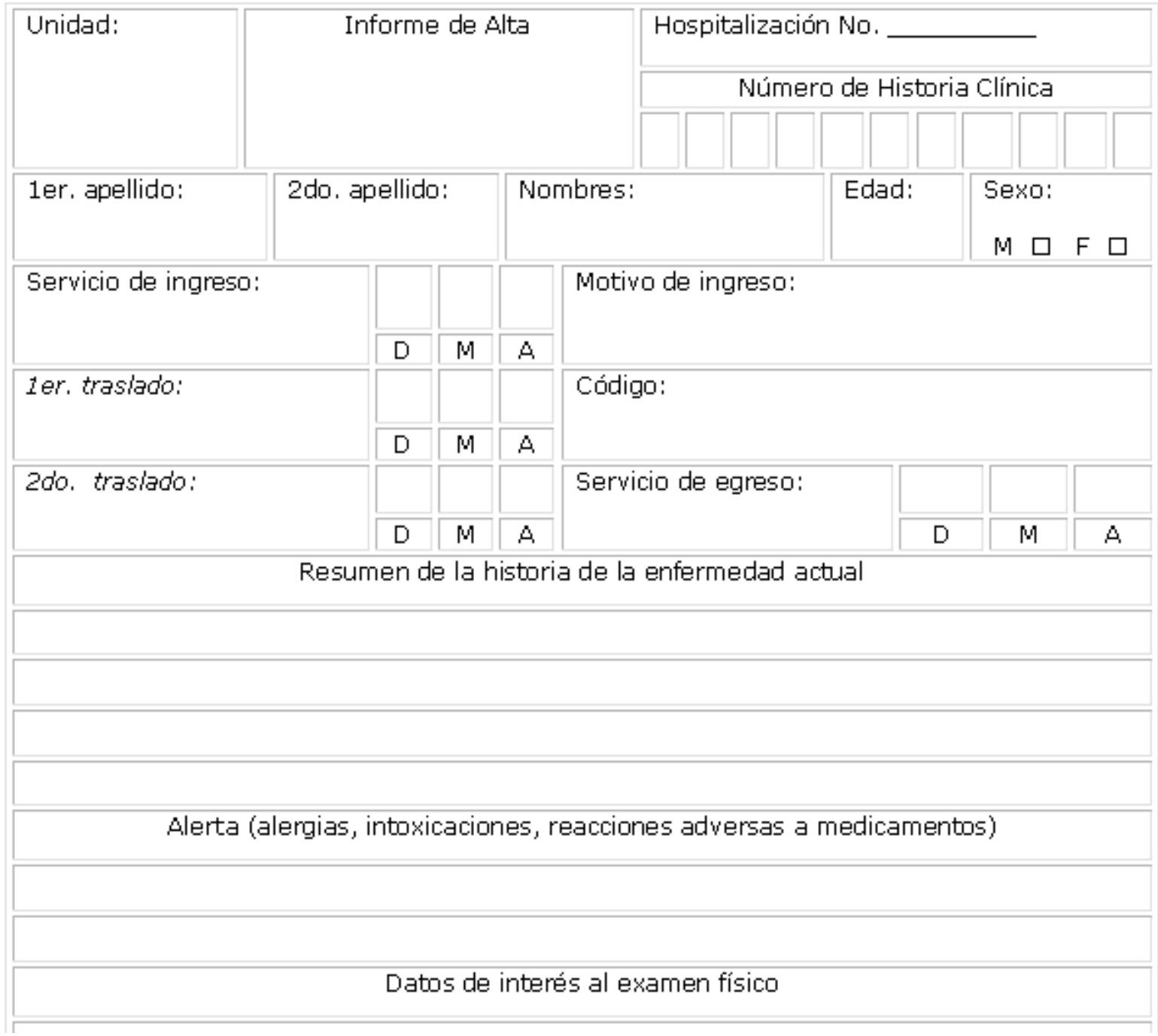


Anotar la palabra "GUARDAR" en los documentos e informes de los exámenes contenidos en la. Historia. Clínica que usted considere de utilidad después de 2 años de realizados

Anotar la cantidad de informes que tienen escrita la palabra "GUARDAR"

\begin{tabular}{|c|c|c|c|}
\hline Tipo de informe & Cantidad & Tipo de informe & Cantidad \\
\hline Laboratorio clínico & & Discusión diagnóstica & \\
\hline Laboratorio de microbiología & & Informe operatorio & \\
\hline Ultrasonido & & Hoja de anestesia & \\
\hline Rayos $x$ & & T.A.C. & \\
\hline Endoscopia & & R.M.N. & \\
\hline Biopsias & & Guardar H.C. de U.C.I. & \\
\hline \multicolumn{4}{|l|}{ Pruebas funcionales } \\
\hline Ecocardiogramas & & & \\
\hline
\end{tabular}

2 años después del alta quedarán en la HC este informe y los que tengan anotados la palabra "GUARDAR"

\begin{tabular}{|c|c|c|}
\hline \multicolumn{3}{|c|}{ Evolución } \\
\hline \multicolumn{3}{|c|}{ Tratamiento (descripción del tratamiento en sala y al alta) } \\
\hline \multicolumn{3}{|c|}{ Para llenar por servicios diferentes al de egreso antes del traslado } \\
\hline \multirow{3}{*}{$\begin{array}{l}\text { Servicio de } \\
\text { terapia }\end{array}$} & Afección principal tratada: & Código: \\
\hline & Ventilado: & APACHE: \\
\hline & Si $\square \quad$ No $\square$ & Al ingreso $\square$ al egreso $\square$ \\
\hline \multirow{3}{*}{$\begin{array}{l}\text { Servicio de } \\
\text { terapia }\end{array}$} & Afección principal tratada: & Código: \\
\hline & Ventilado: & APACHE: \\
\hline & Si $\square \quad$ No $\square$ & Al ingreso $\square$ Al egreso $\square$ \\
\hline \multicolumn{2}{|c|}{ Afección principal tratada: } & Código: \\
\hline
\end{tabular}




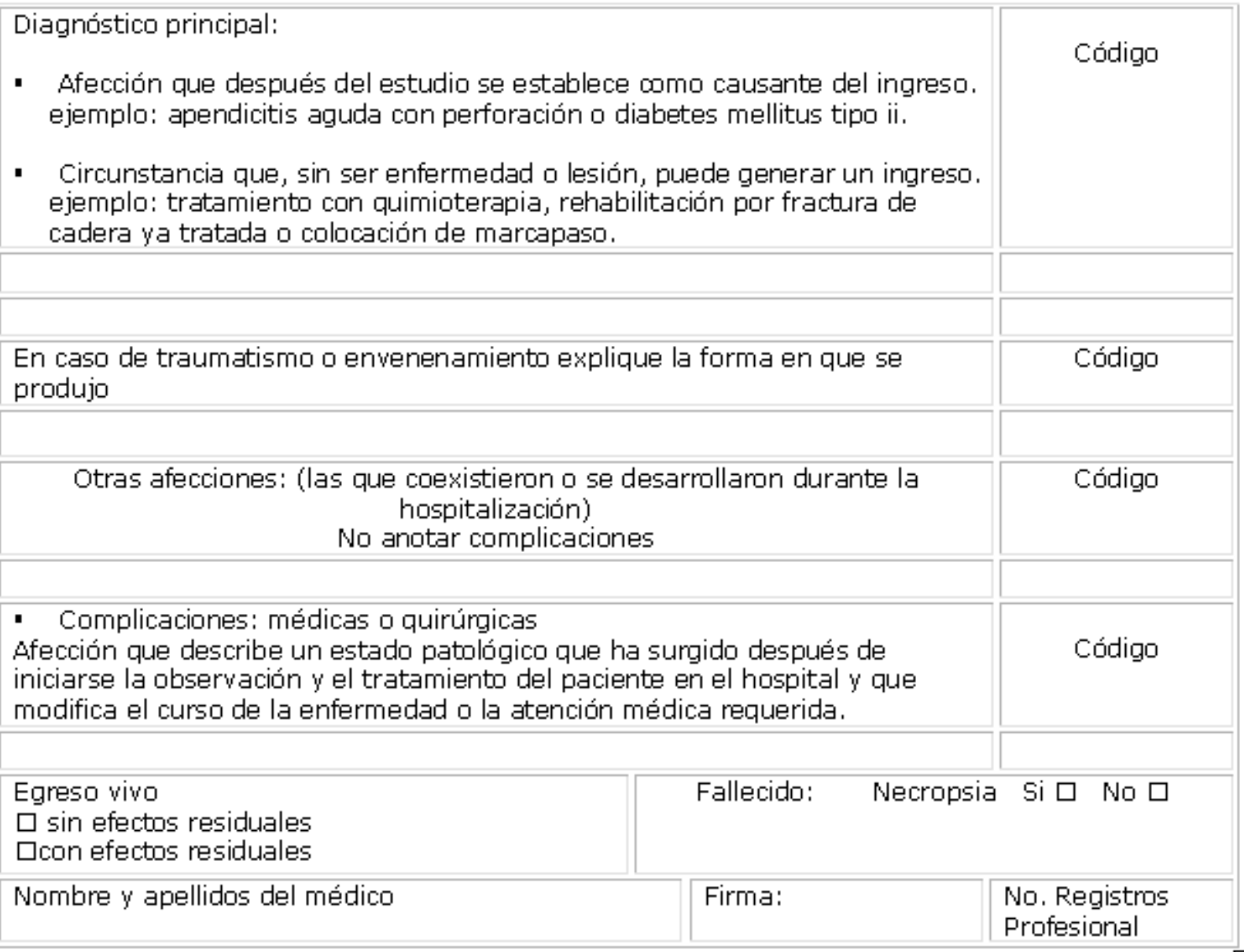

\section{REFERENCI AS BI BLI OGRÁFI CAS}

1. Antolín García González, Celador R. Propuesta de un método de evaluación de la calidad formal del informe médico de alta hospitalaria. España: Todo Hospital; 1997.

2. Casado E, Girón F. Integración de la información del área sanitaria del hospital universitario "San Cecilio" de Granada. Papeles Méd. 2006; (2): 350.

3. Yetano AB, Montero B. Almacenamiento de los informes de alta digitalizados. Papeles Méd. 2002; 66(2): 350.

4. Ríos Massabot NE, Plasencia Iglesias AE, Senra Armas LA, Páez Prats I. El archivo de historias clínicas. Cuestión de Espacio. Rev Cubana Salud Pública. 2002; 28(1): 18-21.

5. Normas de Registros Médicos. La Habana: Dirección Nacional de Estadísticas; 1984.

6. Organización Mundial de la Salud. Clasificación Estadística Internacional de Enfermedades y Problemas Relacionados con la Salud, Décima Revisión. Ginebra: OMS; 1989. [Volumen I - III: XXV]. 
7. Centro Mexicano para la Clasificación de Enfermedades, Procedimientos en Medicina. Clasificación Internacional de Enfermedades 9na. Revisión, modificación clínica 3ra ed. México, D.F: Centro Mexicano (s/f).

Recibido: 11 de noviembre de 2008.

Aprobado: 24 de noviembre de 2008.

María del Carmen Alemán Lage. Hospital Clínico Quirúrgico "Hermanos Ameijeiras". La Habana, Cuba.

E-mail: mariacaleman@infomed.sld.cu 